\title{
BIOPSYCHOSOCIAL IMPLICATIONS RELATED TO THE BREAST CANCER IN WOMEN
}

\author{
Ali Abdulrazig Uossif Alfilani \\ School of Social Sciences, Universiti Sains Malaysia \\ Email Address: alifilani@yahoo.com
}

\begin{abstract}
Keywords: young women, breast cancer, psychological impact, biological consequences, socioeconomic implications.
\end{abstract}

\begin{abstract}
Breast Cancer is among the most common cancer in women. Breast Cancer's aggressiveness truly depends on the age of patient, status of lymph node and size of the tumor. Prognosis may the unfavorable if the age of woman is under 35. Breast cancer, usually, is diagnosed when it is in the advance stage. Looking at all the aspects, this paper analyzes and identifies the socio-economic, psychological and biological implications for the breast cancer in women. In order to meet the objective, the paper applies the qualitative approach and reads all the available literature to find the answers. The results of surveying all the available literature depicts that detection of this cancer at earliest stages is in the benefit of the patient. This benefit is not in case of medical/bio reasons only but also for socio-economic and psychological perspectives. This paper further finds that if the cancer diagnosed in the earliest stages, it not only shorten the hospitalized period but also save a handsome amount of treatment, give satisfactory psychological comforts and a very good life prognosis that results into a rapid familial and social reintegration.
\end{abstract}

\section{INTRODUCTION}

In most of the countries, the common cancer is the breast cancer. For instance, if we look at the European females, the main causes of deaths by cancers are due to breast cancer (Ferlay et al., 2013). Moreover, in Europe in year 2012, 463.8 females out of 0.1 million were suffering from breast cancer, meanwhile the mortality was about 131.2 out of 0.1 million (Ferlay et al., 2013). Biological aggressiveness of the breast cancer depends on many factors, mainly because of age factor. The studies result in better prognosis in ladies who are over 45 years of age, while very unfavorable in the ladies with the age less than 35 years (Aebi et al., 2000). The other important biological factor is the size of tumor. If the tumor is bigger than 2 centimeters, it can leads towards the high risk (Goldrisch et al., 2005). Generally, women diagnose with the breast cancer in the advance stage. If the cancer is detected in the early stages, it can help the patient for the successful surgery with a reduced duration of stay at hospital while a minute adjuvant therapy connected with the periodical outpatient follow-up.

If we look at the psychological factors, if the breast cancer is identified at the early stages, it can give the patient, great psychological comfort and patient can enjoy a rapid family and social insertion. Contrarily, if the cancer is detected at the later stages, adjuvant and neo adjuvant therapy, mammary reconstructions and amputee surgical interventions can leads towards poor life prognosis and other psychological disability factors like lymphedema. Moreover, expensive and aggressive treatments will not allow the patient to labor for the longer time and most probably can lead the patient to go towards the retirement. Consequently, it will decrease the income of the patient that will bring the other socio-economic issues for the patient. Linked with the aspects, a study was conducted on 287 breast cancer patients. It shows that indirect income like lost income and direct cost that is incurred on the health services and other health related expenditures are $80 \%$ more in women that are less than 50 years of their age as compared to the ones that are older than 50 years of age (Gordon et al., 2007). It can be directed that if the breast cancer is detected at the advanced stages, it not only create the physical distress along with the poor life prognosis and also an increased economic and psychological burdens. If the young women are taken into consideration, 
such issues would have disastrous impact considering their relationship with their society and family. Therefore, this paper will identify the biopsychosocial implications for breast cancer.

\section{Literature Review}

Breast cancer is one of the leading reasons of death among the women (Norman \& Brain 2005). Breast cancer can be defined as the growth of malignant cells within the breast tissue. Specifically, it can be occurred due to the results of different tissues under the sway of estrogen infringing and multiplying other tissues, ultimately spreading to the other parts of the body (Boaz 2002).

Breast cancer is precieved as the cruel, chaotic and evil invasion in the body that must be removed (Bahar 2003).

\subsection{The Incidence of Breast Cancer}

In every year the prey of the breast cancer is mostly the ladies with age between 35 to 70 years (Boaz 2002) but it can also affect the younger women. In Australia, breast cancer was the most common cancer (MacDonald et al., 2005). In 2004, 16\% of death due to cancer was because of breast cancer, over 100 men and 11,500 women are being diagnosed with the cancer and nearly 2600 death are due to the breast cancer each year (Breast Cancer Network Australia, 2004) and it accounts for nearly 2,600 deaths annually (National Breast Cancer Foundation, 2005). The overall world statistics shows that 1 lady out of 11 has threat to develop breast cancer in their lives (Women's Health Queensland Wide, 1999a). Some studies also report that young ladies are less likely to have the breast cancer as compare to the older ones. A report shows that out of 10,592 registered cases of breast cancer, only there 684 cases of the women that have the age less than forty years (National Breast Cancer Centre 2004). Therefore, from this we can assume that women in their thirties and twenties are negligible (Umeh \& Rogan-Gibson, 2001). But in younger ladies, breast cancer is more aggressive and advanced as compared to the older ladies (Brennan et al. 2005). Young women having breast cancer have the mortality rate of $72.4 \%$ (National Breast Cancer Centre 2004). These results have been found in all other researches related to breast cancer (National Breast Cancer Centre, 2005).

\subsection{Society's response to breast cancer:}

Because of high mortality and incidence linked with the breast cancer, it is very significant health issues now-a-days (Brewster \& Davidson 2001). The researcher suggests the breast cancer awareness programs to activate and educate the society against the evils of breast cancer. Moreover, scholars noted that self awareness and media awareness campaigns proved to be very effective in raising the awareness of public against the breast cancer. Events such as Breast Cancer day, pink ribbon day and new fashion showing the breast cancer clothing are playing an effective role in educating and spreading public awareness (Chapman et al. 2005). Most of the authors concluded that awareness against the breast cancer was improved (Chan et al., 2007). Chan et al. (2007) found that health van that provided the citizen with the video of breast cancer really improved the awareness. Another research conducted by Jibaja (2000) testified that breast cancer awareness among the women really helped to spread knowledge among the women. Moreover, Howell et al., (2002) focused on increased awareness among college and university students. They measured the increase of awareness in college and university women and compared it with different types of media. The scholars found that students prefer the lectures. Secondly, they found the video to be the most effective followed by the brochures. The scholars, further, found that university women favored the lecture CDs as they allow them to choose and listen to the topic in which they are interested in. The identifications of Howell et al. (2002) are as under

$>$ Include website or phone numbers on material

$>$ Include diverse ethnicity

$>$ Include materials at a variety of places on campus

$>$ Use non-scientific terms 
$>$ Combination of tools more effective

$>$ Education tools should be free of charge

$>$ Portray real women whom they can identify with

All of these mentioned tools were noted to be important to increase awareness; anyhow, different students preferred different types of media over each other.

\subsection{Literature regarding Size of the tumor in breast cancer}

As far as the size of the tumor is concerned, eight studies that include Gajdos et al. (2000), Colleoni et al. (2002), Zabicki et al. (2006), Maurice et al. (2006), Fernandopulle et al. (2006) and Bouchardy et al. (2007) are discussed. The studies concluded that younger women tends to have the larger tumor as compared to the ones with older age.

Three of the six documents that make conclusions regarding size of tumours and age reported younger women tended to have larger tumours (Gajdos et al., 2000; Colleoni et al., 2002; Zabicki et al., 2006). Zabicki et al. (2006) noted that tumor size among all the women has decreased over the time that may result into the early detection. Anyhow, all of them like Maurice et al. (2006) and Fernandopulle et al. (2006) confirm that younger women have larger tumors. In addition, the researchers agreed that young women are mostly affected by the aggressive types of breast cancers (Gajdos et al.,2000, Colleoni et al.,2002, Zabicki et al.,2006, Maurice et al.,2006, Fernandopulle et al.,2006 and Bouchardy et al.,2007)

One research found that $72 \%$ women were detected with breast cancer during their own self examination (Bouchardy et al., 2007). They further points out that $9 \%$ were detected with breast cancer during screening while the other $20 \%$ are diagnosed with breast cancer during the symptoms other than breast cancer.

\subsection{Literature of Breast Cancer in Younger Women}

An immense literature is available on breast cancer in younger women. The literature gives the detailed note on the statistics regarding young women and breast cancer. For example, the SEER report published by United States national Cancer institute in 2007 gives the detailed date of breast cancer patients from 1975 till 2000. They noted that breast cancer among the women aged between 15 to 29 has immensely increased over the passage of time (National Cancer Institute, 2007). Moreover, cases among young adult females and adolescent are also being continuously increased.

\subsection{Suggestion of Treatment}

The study by Trussler (2007) asserts that information regarding breast cancer could be improved as he suggest to focus on specific age groups and education must be focusing on symptoms and signs of cancer and give them guidance over the options of risk reduction. He further recommends that health care facilitators and doctors must focus on supportive and knowledge based practices of breast cancer.

\section{METHODOLOGY FOR THE PAPER}

This section introduces methodology adopted for the study. It describes and justifies use of specific research design and methods employed for the study. Khan (2008) stated that research design is course of entire research process in systematic way. Creswell (2014) maintained that research design shows specific directions for the whole research process. Johnson and Onwuegbuzie (2004) emphasized that research method should be adopted according to nature of research question under study; it increases the possibilities of getting more useful answers. Research Objective (RO) of the current paper is to find the biopsychosocial implications related to the breast cancer in women, therefore, it will be a qualitative research. Morse (1991) stated that researcher adopts qualitative enquiry mode when there is need to explore and describe a phenomenon. Besides, it is qualitative approach is also adopted when given quantitative approach do not suit to study the phenomenon. Moreover, Neuman (2003) stated that when there is little or nothing known about a research question (s), then exploratory research method is undertaken in which researcher intends to 
make new investigation or there is little knowledge available about any question. Therefore, the current study would be exploratory in nature and the study will find the biopsychosoical factors related to the breast cancer. The present paper adopted a system search for the literature available on the biopsychosoical factors related to the breast cancer.

\section{FINDINGS}

Greer et al.1979 is the first psychological and biological assessment of the breast cancer. The researchers assess these factors in breast cancer patients for three and twelve months respectively and then annually collected date from the patients for consecutive four years. All the pateients were conducted with the psychological tests and also interviewed. For the analysis of bio and psycho factors, they designed a social adjustment scale like work record, interpersonal relationship, sexual relations and marital status etc. Moreover, the measured the depression in breast cancer patients by already devised scale by Hamilton, while they checked the hostility by following the questionnaire of hostility designed by Caine and Foulds. In addition, neuroticism and extraversion were calculated by eysnack personality inventory that is called as EPI. For the follow-up examinations, rating of depression and social adjustement were followed systematically and patient's biopsychosocial responses to breast cancer were assessed. The biopsychosocial responses such as hopelessness, helplessness, stoic acceptance, fighting spirit, denial, neuroticism, extroversion, work record, interpersonal relationship, sexual adjustment, psychological stress, depressive illness, hostility, depression, response to stressful events, anger, delay in advice seeking, social class and first response after discovering the breast cancer were assessed. The study found that after the five year of follow-up that $49 \%$ among the selected patients were alive and doing well without any signs of recurrence while $24 \%$ patients were living with metastases but $27 \%$ were died because of this disease and only two patients were died of other disorders. The main finding of the research is that of the patient reacts against the cancer with fighting spirit or denial, recurrence free survival could be seen in them very well. Meanwhile, the patients who behaved with the psycho and social disorders such as hopelessness, stoic acceptance and helplessness suffered from the death.

The second study about the biopsychosocial factors related to breast cancer was conducted by Marshall et al. (1983). During the mentioned study, every patient was inquired about the traumatic actions and events five (5) years before the recognition of different symptoms such as unemployment, divorces, illnesses and death. Apart from that psychological questionnaire, questions related to social involvement such as participation in non-religious and religious activities and marital status etc. Women who were died of breast cancer were included in the study. The variable related to psychology and stress included divorces, unemployment, divorces, illnesses and deaths while social involvement included organization membership, acquaintances, relatives and marital status etc. The outcome of the research concluded that all the patients were died. It concluded that Mean of survival of women $<46$ years in lower and higher stress quartile: 75.8 months and 50.8 months, respectively. Mean of survival of women 45 to 60 years in lower and higher stress quartile: 54 months and 39.2 months, respectively. Mean of survival of women $>61$ years in lower and higher stress quartile: 53.4 months and 54.9 months, respectively. The finding concluded that among all the biopsychosocial characteristics, only predictor of survival against breast cancer is the stage of breast cancer. Moreover, the only significant indicator is social involvement but that is relevant to the patient with the young age only.

The third important study related to biopsychosocial factors related to breast cancer was conducted by Kroenke et al. (2006). Specifically they looked at the social factors related to the breast cancer. Social networks were assessed in 1992, 1996, and 2000 with the Berkman-Syme Social Networks Index (SNI). Social support was assessed in 1992 and 2000 as the presence and availability of confidant. Socialemotional support was assessed in 1992 and 2000 as the presence and availability of a confidant. The factors included social networks such as membership in church and other non-religious organizations and marital status etc. while emotional and support factors included the availability and presence of the confidant and number of communications with $\mathrm{him} / \mathrm{her}$. They found that 107 out of 224 deaths accrue to the year 2004. The pre-diagnostic 
analysis of survival and social networks showed that women who were isolated socially, they had the fear of risk of mortality, $66 \%$ more than others. Apart from that they have two (2) fold increased risk of mortality due to breast cancer as compared to the ones who were socially integrated. Moreover, it was found that women without the children and close relatives had more threat to mortality due to breast cancer and even all cause mortality after the diagnosis if we compare with the ones having strong social bond. Contrarily, participation in community and religious activates was not found to be associated with the survival against breast cancer. The results regarding the post-diagnostic analysis if survival and social networks showed that the less social networks after diagnosis were not totally associated with the survival. But participation in community activities in post-diagnosis examination proved that there appeared to be the low risk of mortality, while, the extent and presence of contact with the confidant found to have no association with the survival against the breast cancer.

The next study is done by Lehto et al. (2006) who have interviewed the patients on the bases of questionnaire called as the WOC i.e. Ways of Coping questionnaire, Life experience survey, Anger expression scale, depression scale and symptoms checklist. They used the factors such as anger expression, emotional expression, perceived available social support, and quality of life after the diagnosis of breast cancer, anger expression and non-cancer life stresses. They measured the survival of breast cancer patients from the day, the patients were diagnosed with the breast cancer up till the date of their death or last follow-up interview. Up till the last data collection, 19.8\% of the patients were died while rest of them had relapsed. They found that behavioral escape avoidance and emotional defensiveness found to be significantly linked with the short survival. As far as the multivariate analysis is concerned, distance coping is linked with the longer survival while high level of perceived support, behavior escaping avoidance coping were found to be linked with the shorter survival. While, depressive symptoms were found to be decreasing the survival chances and high perceived support proved to predict a shorter life.

Osborne et al. (2005) conducted the research on the very main social factor that is marital status and breast cancer. Data were from the Surveillance, Epidemiology and End Results (SEER) tumor registries, merged with Medicare data for the years 1991 through 1995. From the SEER program, information regarding tumor

location, size, axillaries node status, American Joint Committee on Cancer Stage (AJCC), estrogen receptor status, demographic characteristics (age, sex, race, and marital status), and types of treatment provided within 4 months after the date of diagnosis was obtained. The data from the Medicare program used in the study were the Medicare Provider Analysis Review File, the Hospital Outpatient Standard Analytic file, and the 100\% physician/supplier file. Information available through 1998 allowed for 3 years follow-up. The study found that unmarried women had the more risk of mortality from the breast cancer as compared to the ones with marriage and children.

In align with the above mentioned study, Weihs et al. (2005) find the psychosocial issued related to the breast cancer patients. They used the factor to be investigated as the no. of friends and relatives support to the patient and frequency of contacting them. This contact of the patients and their relatives and friends were examined for the 1.6 year after the diagnosis of breast cancer in the women. All the available women of stage 2 and stage 3 were contacted. At the time of enrollment, from 1991 until 1993, the patients were examined for disease and support severity using

Nottingham Prognostic Index (NPI) and afterwards monitoring was done until the December, 2000. Participants listed the names of their friends and relatives and told about the frequency of contacting them. Apart from that, for looking at the disease aggressiveness, the annual medical charts were assessed. The result found the 1 standard deviation increase in the support of dependable may lead to decrease in the mortality rate by 1 standard deviation.

Meanwhile another very important study by Osborne et al. (2004) studied the biopsychosocial factors of breast cancer patients that included the social support, adjustment to cancer, depression and anxiety. The methodology adopted included Mental Adjustment to Cancer (MAC), Duke-UNC Functional Social Support (DUFS) and Hospital Anxiety and Depression Scale (HADS). They did the follow-up interviews minimum for about 6.1 years and maximum for 7.9 years. The data was 
collected from the date of registration till the death of patient or the lase follow-up. From the results, they showed that $29 \%$ patients were died while $5 \%$ died because of other reasons.

The study finds that fighting spirits of the patient helped to minimize the level of illness and helped in long survival. Social support, depression and anxiety did not significantly the survival of the patients. Further, it was noted that leukocyte numbers were weakly associated with depression and helplessness. As far as the biological factors are concerned, no substantial and considerable proof of psycho-neuro-immunological was seen between biological factors and psychosocial factors.

Another important study was conducted by Soler-Vila et al. (2005) who researched about the curability detection and treatment of cancer keeping the biopsychosocial factors and different believes about the breast cancer. Among all the selected patients, $90 \%$ of the patients were interviewed during the six months of their diagnosis with the disease while remaining $10 \%$ were conducted within 1 year after the detection of the breast cancer. The information was collected through white cancer survival study that gather data on the biopsychosocial factors related to the breast cancer. The study found that at the end of year 2002, breast cancer was the reason of about $66 \%$ death.

The last mentioned study was conducted by Graham et al. (2002). They conducted the research on long standing difficulties and discrete life events associated with the breast cancer. They collected the data on depression and stressful experience from breast cancer patient through interviews and also recorded them. The time period for data collection was 18 months that collected the data from the patients suffered from breast cancer covering the period from 12 month $\mathrm{s}$ to 5 years after the detection of breast cancer among them. They adopted the structured clinical interview protocol. They found that No evidence that women who have a severely stressful life experience in the year before breast cancer diagnosis, or in the 5 years following are at any increased risk of developing a recurrence of their disease. An episode of depression before diagnosis did not increase the risk of recurrence (hazard ratio 1.22 , CI 0.38 to $3.92, p=0.7$ ). No increased risk of recurrence in women who had had one or more severely stressful experiences in the year before diagnosis compared with women who did not (hazard ratio 1.01, CI

0.58 to $1.74, p=0.99$ ). In the post-diagnosis period, women who had one or more severely stressful life experiences had a lower risk or recurrence than those who did not (hazard ratio 0.52, CI 0.29 to $0.95, \mathrm{p}=0.03$ )

\section{DISCUSSIONS \& CONCLUSION}

The paper after looking at the biopsychosocial implications related to the breast cancer in women concludes that if the breast cancer is identified at the early stages, it can give the patient, great psychological comfort and patient can enjoy a rapid family and social insertion. Contrarily, if the cancer is detected at the later stages, adjuvant and neo adjuvant therapy, mammary reconstructions and amputee surgical interventions can leads towards poor life prognosis and other psychological disability factors like lymphedema. Moreover, expensive and aggressive treatments will not allow the patient to labor for the longer time and most probably can lead the patient to go towards the retirement. Consequently, it will decrease the income of the patient that will bring the other socio-economic issues for the patient. It can be directed that if the breast cancer is detected at the advanced stages, it not only create the physical distress along with the poor life prognosis and also an increased economic and psychological burdens. If the young women are taken into consideration, such issues would have disastrous impact considering their relationship with their society and family. Considering the results, it can be rightly concluded that if the detection of cancer is done at the early stages, it helps the patient from socio-economic, psychological and biological/medical point of view. Unluckily, if it is diagnosed at the advance stages, Biopsychosocial factors don't help the patients a lot. 


\subsection{Conclusion}

The results of surveying all the available literature depicts that detection of this cancer at earliest stages is in the benefit of the patient. This benefit is not in case of medical/bio reasons only but also for socio-economic and psychological perspectives. This paper further finds that if the cancer diagnosed in the earliest stages, it not only shorten the hospitalized period but also save a handsome amount of treatment, give satisfactory psychological comforts and a very good life prognosis that results into a rapid familial and social reintegration. The literature suggests that to explore the vulnerability to cancer against women, there is a need of better systematic understanding of the effects of rising up in the family prone to breast cancer. Apart from that there is an insight need to know the family relationships. Improved methods of risk communication will also be important, to ensure that women have appropriate risk information with which to make informed choices about risk management options and preventative interventions.

\section{References}

[1] Aebi, S., Gelber, S., Castiglione-Gertsch, M., Gelber, R.D., Collins, J., Thurlimann, B. et al. (2000). Is chemotherapy alone adequate for young women with oestrogen receptor positive breast cancer? Lancet, 355(9218), 1869-1874.

[2] Anghel, R., Minea, L., Dediu, M., Bacinschi, X., Eniu, A., Eniu, D., Miron, L. \& Aldea,B. (2009). Ghid de management al cancerului mamar. Ordinul ministrului $s \backslash n \backslash t \backslash]$ ii no. 1059, Annex 9, Monitorul Oficial, part I no. 608bis.

[3] Beckman, J., Johansen, L., Richardt, C. \& Blickert-Toft, M. (1983). Psychological reactions in younger women operated on for breast cancer. Danish Medical Bulletin,30(2), 10-13.

[4] Bigatti, S.M., Steiner, J.L., Makinabakan, N., Hernandez, A.M., Johnston, E. and Storniolo,A.M. (2012). Matched and mismatched cognitive appraisals in patients with breast cancer and their partners: implications for psychological distress. Psycho-Oncology, 21(11), 1229-1236.

[5] Cormier, J.N., Askew, R.L., Mungovan, K.S., Xing, Y., Ross, M.I. \& Armer, J.M. (2010).Lymphedema beyond breast cancer: a systematic review and metaanalysis of cancerrelated secondary lymphedema. Cancer, 116(22), 5138-5149.

[6] Deadman, J.M., Dewey, M.J., Owens, R.G., Leinster, S.J. \& Slade, P.D. (1989). Threat and loss in the breast cancer. Psycho-Social Medicine, 19, 677-681.

[7] Dean, C., Chetty, U. \& Forrest, A.P.M. (1983). Effects of immediate breast reconstrusction on psychosocial morbidity after mastectomy. Lancet, 1, 459-462.

[8] Diaconu, C., Livadariu, R.M. \& Dogaru, C. (2012). The risk of lymphedema after breast cancer surgical treatment. The Medical Surgical Journal, 116(4), 1081-1086.

[9] Ernstmann, N., Neumann, M., Ommen, O., Galushko, M., Wirtz, M., Voltz, R., Hallek, M. \& Pfaff, H. (2009). Determinants and implications of cancer patients' psychosocialneeds. Supportive Care in Cancer, 17(11), 1417-1423.

[10] Ferlay, J., Steliarova-Foucher, E., Lortet-Tieulent, J., Rosso, S., Coebergh, J.W.W., Comber, H., Forman, D. \& Bray, F. (2013). Cancer incidence and mortality patterns in Europe: Estimates for 40 countries in 2012, European Journal of Cancer, 49,1374-1403.

[11] Goldrisch, A., Glick, J.H., Gelber, R.D., Coates, A.S., Thurlimann, B. \& Senn, H.J. (2005). Meeting highlights: International expert Consensus on the primary therapy of early breast cancer. Annals of Oncology, 16, 1569-1583.

[12] Gordon, L., Scuffham, P., Hayes, S. \& Newman, B. (2007). Exploring the economic impact of breast cancers during the 18 months following diagnosis. Psycho-Oncology, 16(12), 11301139.

[13] Harmer, V. (2006). The psychological implications of breast cancer. Practice Nurse,32(6), 4449.

[14] Hewitt, M., Herdman. R. \& Holland, J. (2004). Meeting psychosocial needs of women with breast cancer, Washington: The National Academies Press. 
[15] Holland, J.C. (1998). Societal views of cancer and the emergence of psychooncology. In Holland, J.C. (ed.), Psychooncology, New York: Oxford University Press, pp. 3-15.

[16] Greer, S., Morris, T., \& Pettingale, K. W. (1979). Psychological response to breast cancer: effect on outcome. The Lancet, 314(8146), 785-787.

[17] Marshall, J. R., \& Funch, D. P. (1983). Social environment and breast cancer. A cohort analysis of patient survival. Cancer, 52(8), 1546-1550.

[18] Kroenke, C. H., Kubzansky, L. D., Schernhammer, E. S., Holmes, M. D., \& Kawachi, I. (2006). Social networks, social support, and survival after breast cancer diagnosis. Journal of Clinical Oncology, 24(7), 1105-1111.

[19] Lehto, U. S., Ojanen, M., Dyba, T., Aromaa, A., \& Kellokumpu-Lehtinen, P. (2006). Baseline psychosocial predictors of survival in localised breast cancer.British journal of cancer, 94(9), 1245-1252.

[20] Osborne, C., Ostir, G. V., Du, X., Peek, M. K., \& Goodwin, J. S. (2005). The influence of marital status on the stage at diagnosis, treatment, and survival of older women with breast cancer. Breast cancer research and treatment, 93(1), 41-47.

[21] Weihs, K. L., Enright, T. M., Simmens, S. J., \& Reiss, D. (2000). Negative affectivity, restriction of emotions, and site of metastases predict mortality in recurrent breast cancer. Journal of psychosomatic research, 49(1), 59-68.

[22] Osborne, R. H., Sali, A., Aaronson, N. K., Elsworth, G. R., Mdzewski, B., \& Sinclair, A. J. (2004). Immune function and adjustment style: do they predict survival in breast cancer?. Psycho-Oncology, 13(3), 199-210.

[23] Soler-Vila, H., Kasl, S. V., \& Jones, B. A. (2003). Prognostic significance of psychosocial factors in African-American and white breast cancer patients.Cancer, 98(6), 1299-1308.

[24] Graham, J., Ramirez, A., Love, S., Richards, M., \& Burgess, C. (2002). Stressful life experiences and risk of relapse of breast cancer: observational cohort study. Bmj, 324(7351), 1420. 